\title{
The deficiency of FKBP-5 inhibited hepatocellular progression by increasing the infiltration of distinct immune cells and inhibiting obesity-associated gut microbial metabolite
}

\author{
Chuantao Zhang ${ }^{1 \#}$, Xiang Cui ${ }^{2 \#}$, Lian Feng ${ }^{3}$, Zhiyi Han ${ }^{2}$, Deti Peng ${ }^{2}$, Wenjun Fu ${ }^{4}$, Yufeng Xing ${ }^{2}$ \\ ${ }^{1}$ Department of Respiratory Medicine, Hospital of Chengdu University of Traditional Chinese Medicine, Chengdu, China; ${ }^{2}$ Department of \\ Hepatology, Shenzhen Traditional Chinese Medicine Hospital, Shenzhen, China; ${ }^{3}$ The Fourth Clinical Medical College of Guangzhou University of \\ Chinese Medicine, Shenzhen, China; ${ }^{4}$ South China Research Center for Acupuncture and Moxibustion, School of Basic Medical Science, Guangzhou \\ University of Chinese Medicine, Guangzhou, China \\ Contributions: (I) Conception and design: C Zhang, X Cui, W Fu, Y Xing; (II) Administrative support: Z Han, D Peng, W Fu; (III) Provision of \\ study materials or patients: C Zhang, Z Han, W Fu; (IV) Collection and assembly of data: C Zhang, X Cui, Z Han, D Peng; (V) Data analysis and \\ interpretation: C Zhang, X Cui; (VI) Manuscript writing: All authors; (VII) Final approval of manuscript: All authors. \\ \#These authors contributed equally to this work. \\ Correspondence to: Wenju Fu. Guangzhou University of Chinese Medicine, No. 232, East Waihuan Road, Guangzhou University City, Panyu District, \\ Guangzhou 510006, China. Email: fuqingzhu2006@163.com; Yufeng Xing. Shenzhen Traditional Chinese Medicine Hospital, No. 1, Fuhua Road, \\ Futian District, Shenzhen 518033, China. Email: yufeng000729@163.com.
}

Background: Gut microbiota has a number of essential roles in nutrition metabolism and immune homeostasis, and is closely related to hepatocellular progression. In recent years, studies have also shown that FK506 binding protein 5 (FKBP-5) plays a crucial role in immune regulation. However, it is not yet clear whether FKBP-5 promotes the development of hepatocellular carcinoma (HCC) by affecting immune function and gut microbiota.

Methods: FKBP-5 expression was verified by immunochemistry and western blot and reverse transcription polymerase chain reaction (RT-qPCR) assays. After treatment in WT and $\mathrm{FKBP}-5^{-/-}$mice, the histological characteristic of mice liver tissue was assessed by H\&E staining, and hepatic leukocytes and hepatic NKT cells were identified by flow cytometer. Meanwhile, primary bile acids (BAs), secondary BAs, serum total cholesterol, and the weight of abdomen adipose tissues were examined, and the gut microbiota was evaluated by $16 \mathrm{~S}$ ribosomal ribonucleic acid (rRNA) sequencing.

Results: We discovered that FKBP-5 was highly expressed in HCC tissues. Meanwhile, FKBP-5 deletion inhibited tumor progression by increasing $\mathrm{CD}^{8+} \mathrm{T}, \mathrm{CD}^{4+} \mathrm{T}, \mathrm{NKT}$ and $\mathrm{CD}^{4+} \mathrm{NKT}$ cells in mice after diethylnitrosamine (DEN) injection. Besides, we proved that FKBP-5 deletion generated rapid and significant reductions in the intestinal BAs, the weight of abdomen adipose tissues and the serum total cholesterol. FKBP-5 deletion also led to a change in the composition of gut microbiota, suggesting that BAs are the main dietary factor regulating gut microbiota, which could be affected by FKBP-5 deletion. Further, we uncovered that anti-CD4 and anti-CD8 treatments facilitated hepatocellular progression by modulating gut microbiota composition in $\mathrm{FKBP}-5^{-/-}$mice.

Conclusions: Therefore, we demonstrated that FKBP-5 deletion inhibited hepatocellular progression by modulating immune response and gut microbiome-mediated BAs metabolism.

Keywords: FK506 binding protein 51; hepatocellular carcinoma (HCC); immune cells; gut microbiota; bile acids

Submitted Dec 23, 2020. Accepted for publication Apr 12, 2021.

doi: 10.21037/jgo-21-71

View this article at: http://dx.doi.org/10.21037/jgo-21-71 


\section{Introduction}

Hepatocellular carcinoma (HCC) is a malignant cancer, and has the sixth highest incidence rate and the third highest mortality rate among all cancers worldwide (1). In 2012, an estimated 780,000 people were diagnosed with HCC, and 750,000 died of HCC worldwide (2). More than $50 \%$ of the new and fatal cases of HCC occurred in China (3). HCC develops from long-term chronic liver diseases (LTCLD), such as alcoholic liver diseases (ALD), non-alcoholic fatty liver diseases (NAFLD), and repeated injury, inflammation and regeneration of hepatocytes caused by viral hepatitis $(1,2)$. Intestinal flora is a complex and dynamic microecosystem in the human digestive tract, which has a balanced symbiotic relationship with the host under physiological conditions (4). In recent years, research has repeatedly shown that the composition and function changes of the symbiotic microbial community in the human body are closely related to various diseases and health conditions (5). One study demonstrated that intestinal microbes are associated with tumor formation (6). Intestinal microorganisms can lead to the occurrence of tumors by integrating oncogenes into the host genome, affecting the stability of the host genome, and breaking the balance between the host immune system (6). Recent studies have shown that intestinal leakage, gut microbiota imbalances, microbe-associated molecular patterns and microbial metabolites may promote the development of HCC by affecting liver inflammation, increasing cell proliferation and inhibiting anti-tumor immunity $(7,8)$. Besides, changes in the gut microbial composition and intestinal permeability can result in the imbalance of gut microbial and bacterial migration (9). The gut microbial and their metabolites can not only enter the liver to activate inflammation-related signaling pathways, but also enter the systemic circulation to form endotoxemia, jointly promoting the development of chronic liver disease and HCC $(10,11)$. However, the specific mechanism by which gut bacteria contribute to the progression of liver cancer remains unclear.

The FK506 binding protein 5 (FKBP-5), also known as FKBP-51, is a FK506 binding protein (12). FKBP-5 has two peptidyl-prolyl cis/trans isomerases domains and tree polypeptide repeat (TPRs) domains that mediate proteinprotein interactions (13). FKBP-5 can directly bind to heat shock protein (HSP90) and participate in the formation and functional regulation of complex steroid hormone receptors through its TPR domain $(14,15)$. Many investigations have suggested that FKBP-5 can regulate plentiful biological processes, including immune regulation, steroid receptor activation, cell survival, protein synthesis, and folding and transportation (16-18). In recent years, FKBP-5 has also been found to have significant effects in the development of tumors (19-22). FKBP-5 has been found to be highly expressed in melanoma, prostate cancer, esophageal adenocarcinoma, glioma and other tumor tissues, and closely related to cell invasion, metastasis, and tumor drug resistance (23-25). However, the function and mechanism of FKBP-5 on HCC progression have not yet been fully elucidated.

At present, research has shown that as a negative regulator of glucocorticoid receptor, FKBP-5 plays an essential role in regulating glycolipid metabolism (26-28). For example, FKBP-5 knockout mice can resist obesity induced by a high-fat diet by enhancing autophagy (29). In addition, studies have also demonstrated that gut microbiota regulates human glucose and lipid metabolism by inhibiting Adenosine 5'-monophosphate (AMP)-activated protein kinase, inducting adipocytokine activity, regulating the activation of nuclear receptor peroxisome proliferatoractivated receptor- $\gamma$ (PPAR- $\gamma$ ), and changing intestinal permeability $(30,31)$. Thus, it was hypothesized that FKBP5 might change the composition of gut microbiota.

In the present study, we aimed to explore the biological functions and underlying molecular mechanisms of FKBP5 in HCC. Specifically, we identified the expression level of FKBP-5 in HCC. After diethylnitrosamine (DEN) injections, we conducted experiments to evaluate the effects of FKBP-5 deletion on the recruitment and activation of lymphocytes in HCC. We also investigated the concentration of intestinal bile acids (BAs), the alteration of gut microbiome, and the development of obese phenotypes. Our results showed that anti-CD4 and anti-CD8 treatments promote hepatocellular progression by modulating gut microbiota composition in $\mathrm{FKBP}-5^{-/-}$mice.

We present the study in accordance with the ARRIVE reporting checklist (available at http://dx.doi.org/10.21037/ jgo-21-71).

\section{Methods}

\section{Patients and sample collection}

HCC tissues and matched para-carcinoma tissues $(5 \mathrm{~cm}$ from the margin of cancer) were extracted from HCC patients at the Shenzhen Traditional Chinese Medicine Hospital from January 2018 to October 2018. The pathological features of the tissues were identified by an experienced pathologist. During the operation, the patients' 
tissues were extracted and immediately stored in a $-80{ }^{\circ} \mathrm{C}$ refrigerator. The study was conducted in accordance with the Declaration of Helsinki (as revised in 2013). Written and informed consent forms were obtained from all of the participating patients. The study was approved by the Shenzhen Traditional Chinese Medicine Hospital Ethics Committee.

\section{Immunochemistry assay}

Adopting the approach of previous research (32), HCC tissues and para-carcinoma tissues were dewaxed and hydrated. The peroxidase activity was blocked through the usage of $3 \%$ hydrogen peroxide, and ethylene diamine tetraethyl acid was applied at a high temperature and high pressure for the antigen retrieval. The sections were blocked with $10 \%$ goat serum (Life Technologies, cat. no. 16210-064) for 40 minutes at $37{ }^{\circ} \mathrm{C}$ and incubated with primary antibody (FKBP-5, Abcam, ab2901) overnight at $4{ }^{\circ} \mathrm{C}$. The next day, after washing with phosphate-buffered saline (PBS), the sections were disposed with goat antirabbit IgG H\&L (HRP, Abcam, ab6721) for 20 minutes at $37^{\circ} \mathrm{C}$. After treatment with 3, 3'-diaminobenzidine (R\&D Systems, cat. no. CTS010), the nuclei were stained with hematoxylin (Sigma-Aldrich, cat. no: HHS32). The results were observed using a microscope.

\section{Western blot assay}

As discussed previously (33), the extracted protein $(30 \mu \mathrm{L})$ was isolated using SDS-PAGE and transferred to nitrocellulose membranes (Millipore, Billerica, MA, USA). After blocking with 5\% low-fat dried milk for 2 hours, the membranes were incubated with primary antibodies against anti-human FKBP-5 (1/1,000, Abcam, Cambridge, UK, ab2901) and GAPDH (1/2,000, Abcam, ab128915) overnight at $4{ }^{\circ} \mathrm{C}$. After washing with PBS, a secondary antibody $(1 / 2,000$, Abcam, ab205718) was used to incubate the membranes for 2 hours at room temperature. Finally, the results were examined using enhanced chemiluminescent reagents.

\section{Cell culture}

Human liver (HL)-7702 and HCC cells (i.e., SMMC7721, Hep 3B, Huh7, Hep G2, and LO2) were purchased from the American Type Culture Collection. HL-7702, SMMC-7721, Hep 3B, Huh7, and Hep G2 were grown in the Dulbecco's Modified Eagle's medium (Hyclone), and LO2 cells were incubated in RPMI 1640 medium (Gibco, Grand). 10\% fetal bovine serum (Gibco) and $1 \%$ streptomycin-penicillin (Invitrogen) were added to all media. All cells were cultured at $37^{\circ} \mathrm{C}$ in a humidified incubator with $5 \% \mathrm{CO}_{2}$.

\section{Reverse transcription quantitative polymerase chain reaction assay}

Total ribonucleic acid (RNA) was extracted using a TRIzo Reagent (Invitrogen, cat. no. 15596026) in accordance with the directions provided. A NanoDrop 2000 Spectrophotometer (Thermo Scientific) was also applied to evaluate the quality of the RNA. The first strand complementary deoxyribonucleic acids (DNAs) were synthesized using a PrimeScript RT reagent kit (Takara, Tokyo, Japan) with $1 \mu \mathrm{g}$ RNAs. Next, polymerase chain reaction (PCR) amplification was used to examine the FKBP-5 level using a SYBR ${ }^{\circledR}$ Premix Ex Taq ${ }^{\mathrm{TM}}$ II kit (TaKaRa, cat. no. RR820A) on an Applied Biosystems 7500 Fast Real-Time PCR system. The relative expression level of FKBP-5 was counted using the $2^{-\Delta \Delta c t}$ method. The sequences of primers were as follows: mouse FKBP-5, forward primer: 5'-CCCCAATGCTGAGCTTATGT-3', reverse primer: 5'-AACGACTCTGAGGCTTTGGA-3'; human FKBP-5, forward primer: 5'-TCCCTCGAATGCAACTCTCT-3', reverse primer: 5'-AAACATCCTTCCACCACAGC-3'; mouse $\beta$-actin, forward primer: 5 '-TATGCCAACACAG T G T T G T C T G G-3 ', rever s e primer : 5'-TACTCCTGCTTGCTGATCCACAT-3'; human $\beta$-actin, forward primer: $5^{\prime}$-ATCTGGCACCACACCTTC TACAATGAGCTGCG-3', reverse primer: 5'-CGTCATA CTCCTGCTTGCTGATCCACATCTGC -3'.

\section{Animals}

FKBP-5 ${ }^{-/}$C57BL/6 mice and wild-type (WT) C57BL/6 mice were provided by the Institute of Experimental Animals, Academy of Medical. Sciences of China. We chose FKBP$5^{-/-}$mice and WT mice (age: 8 weeks, weight: $20-24 \mathrm{~g}$; there were 5 mice in each group). The animals were bred to adapt to the new environment. The mice lived in comfortable environments (were provided with adequate and clean food and water, and exposed to 12 hours of lighting per day in an environment with 50-60\% humidity and temperatures of $18-22{ }^{\circ} \mathrm{C}$ ). The program for the animal 
experiment was approved by the Institutional Animal Care and Use Committee. In the process of the experiment, the principle of animal welfare was fully considered.

\section{Treatment}

At 14 days, an intraperitoneal injection of $25 \mathrm{mg} / \mathrm{kg}$ diethylnitrosamine (DEN, Sigma-Aldrich, Munich, Germany) was used to induce liver cancer. At 10 weeks, the mice were treated with anti-CD4/anti-CD8, and at 25 weeks, the mice were executed.

\section{Measures of intrabepatic leukocytes}

According to previous research (34), intrahepatic leukocytes were separated and assessed using fluorescence-activated cell sorting (FACS).

\section{Hematoxylin and eosin staining}

The live tissues from the WT mice, $\mathrm{FKBP}-5^{-1-}$ mice and anti-CD4 and anti-CD8-treated $\mathrm{FKBP}-5^{-/-}$mice were fixed in $4 \%$ paraformaldehyde (Sigma-Aldrich, cat. no. P6148) and dehydrated with alcohol. Next, 5- $\mu$ m sections were stained with hematoxylin and eosin (H\&E) solution (Solarbio, H8070). The results were examined using a microscope (Nikon, Japan).

\section{$16 S$ ribosomal ribonucleic acid sequencing}

The contents of the rectal segments of the mice in each group were collected, and DNAs were extracted using a PowerSoil DNA Isolation Kit (MO-BIO, cat. no. 12888) in accordance with the experimental instructions provided. RNA amplification was performed using Q5 High-Fidelity 2x Master Mix (New England Biolabs) of the extracted DNAs. After RNA purification, high-throughput sequencing was performed to analyze the sequences of $16 \mathrm{~S}$ using an Illumina MiSeq platform. The complete obtained genetic sequences were uploaded to MG-RAST. After comparing these to the Greengenes database, the characteristics of the bacterial community structure were obtained.

\section{BAs and serum total cholesterol detection}

The levels of gut BAs were analyzed using the BA Assay Kit by Enzyme Cycle Method (Sichuan Maker Biotechnology Co., Ltd. Chengdu, China; CH0103005 R1) in accordance with the instructions provided. The levels of serum total cholesterol were also assessed using a total Cholesterol Test Kit (cat. no. 58236) according to the experimental instructions provided. In addition, the weight of the abdomen adipose tissues was measured.

\section{Statistical analysis}

In this study, data were expressed as mean \pm standard error of the mean (SEM). The results were analyzed using SPSS v20 software (IBM Corp., Armonk, NY, USA), and a oneway analysis of variance was conducted. Differences in abundance were evaluated using a non-parametric factorial Kruskal-Wallis sum-rank test based on LEfSe software. A classification analysis was undertaken using the Ribosomal Database Project (Classifier). A $\mathrm{P}<0.05$ was considered statistically significant.

\section{Results}

\section{FK506 binding protein 5 was highly expressed in HCC}

To analyze the expression level of FKBP-5 in HCC, HCC tissues and para-carcinoma tissues were collected. The results of the immunochemistry (IHC) assay showed that FKBP-5 expression was higher in HCC tissues than the matched para-carcinoma tissues (see Figure $1 A$ ). Similarly, the results of the western blot assay demonstrated that FKBP-5 was more highly upregulated in HCC tissues than para-carcinoma tissues (see Figure 1B). Additionally, we found that compared to HL-7702 cells, the level of FKBP5 was significantly aggrandized in HCC cells (i.e., Hep G2, SMMC-7721, Hep 3B, Huh7, and LO2) $(\mathrm{P}<0.05$, $\mathrm{P}<0.001$; see Figure 1C). Thus, we proved that FKBP-5 was upregulated in HCC.

\section{The deficiency of FK506 binding protein 5 inbibited DEN- induced hepatocellular progression}

To verify that tumor progression was regulated by immune cells but not by parenchyma or other nonparenchymal cells, WT and $\mathrm{FKBP}-5^{-/-}$mice were intraperitoneally injected with DEN at 14 days, and mice were executed at 25 weeks (see Figure 2A). The results of the H\&E staining showed that the number and growth of liver tumors were lower in FKBP- $5^{-1-}$ mice than in WT mice $(\mathrm{P}<0.05$; see Figure $2 B)$. Notably, the messenger ribonucleic acid (mRNA) expression level of FKBP-5 increased dramatically after DEN treatment in WT mice at 25 weeks $(\mathrm{P}<0.05$; see 
A

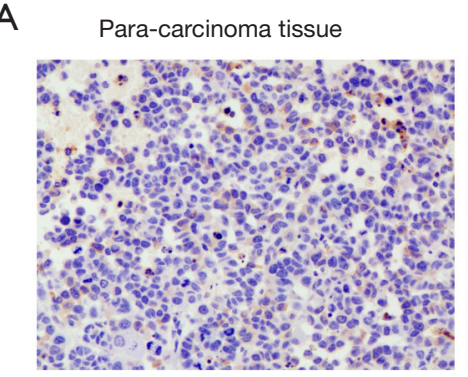

B

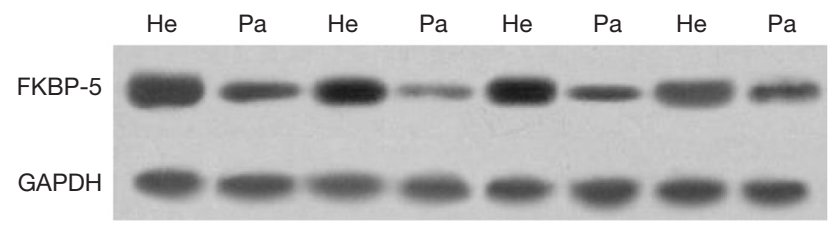

He: Hepatocellular carcinoma tissue

Pa: Para-carcinoma tissue
Hepatocellular carcinoma tissue

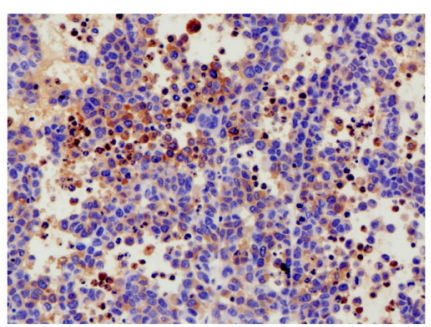

C

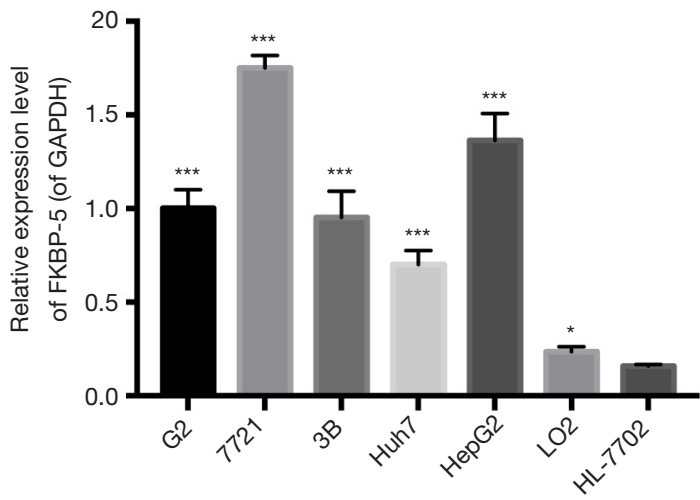

Figure 1 FKBP-5 was highly expressed in HCC. (A) The expression of FKBP-5 was examined by the IHC staining of para-carcinoma tissues and HCC tissues. The representative images were displayed. Magnification, 100x; Scale bar $=100 \mu \mathrm{m}$. (B) The western blotting analysis of FKBP-5 expression in HCC tissues (He) and para-carcinoma tissues (Pa), GAPDH was used as an internal reference. (C) The mRNA expression level of FKBP-5 was confirmed by RT-qPCR assay in HL-7702 and five HCC cells (SMMC-7721, Hep 3B, Huh7, Hep G2, and LO2), *, $\mathrm{P}<0.05 ;$ ***, $\mathrm{P}<0.001$ vs. HL-7702 cells.

Figure 2C). Further, FACS gating was used to analyze the characteristics of the hepatic leukocyte populations. As Figure $2 D$ shows, the proportions of hepatic leukocytes $\left(\mathrm{CD}^{+}{ }^{+} \mathrm{T}\right.$ cells, $\mathrm{CD} 4^{+} \mathrm{T}$ cells, NKT cells) and hepatic NKT $(\mathrm{CD} 4+\mathrm{NKT})$ cells were significantly higher in $\mathrm{FKBP}-5^{-1-}$ mice than WT mice $(\mathrm{P}<0.05, \mathrm{P}<0.001)$. Thus, we suggested increasing the infiltration of distinct immune cells to knock out FKBP-5 inhibited hepatocarcinogenesis.

\section{FK506 binding protein 5 deletion-mediated gut microbial alterations were related to $B A s$}

To further demonstrate the interaction of BAs with gut microbiota, the concentration of BAs was assessed in the gut content of WT and FKBP- $-5^{-/-}$mice. The results showed that the $\mathrm{FKBP}-5^{-/-}$mice had markedly lower levels of gut BAs than WT mice $(\mathrm{P}<0.05, \mathrm{P}<0.001$; see Figure $3 A)$. Our results also showed that FKBP-5 deletion significantly decreased the weight of abdomen adipose tissues and the level of serum total cholesterol, which suggests a correlative relationship between FKBP-5 deletion and phenotype alteration $(\mathrm{P}<0.001$; see Figure $3 A)$. In addition, the results from the phylum bar plot showed that the composition of gut microbiota was similar in WT and $\mathrm{FKBP}-5^{-1-}$ mice, and the abundance of bacteroidales and verrucomicrobiales and clostridiales, burkbolderiales and enterobacteriales were higher in $\mathrm{FKBP}-5^{-/-}$mice than WT mice (see Figure 3B). Thus, FKBP-5 deletion may reduce gut microbiome alterations and the development of obese phenotypes.

\section{The combination of anti-CD4 and anti-CD8 promoted bepatocellular progression by modulating gut microbial community in FK506 binding protein $5^{-1-}$ mice}

Next, we explored the roles of anti-CD4 and anti-CD8 on hepatocellular progression in $\mathrm{FKBP}-5^{-/-}$mice. As Figure $4 A$ shows, at day $14, \mathrm{WT}$ and $\mathrm{FKBP}-5^{-1-}$ mice were intraperitoneally injected with DEN, at 10 weeks, mice were treated with anti-CD4/anti-CD8, and at 25 weeks, mice were executed. We found that the number and growth of liver tumors were higher in anti-CD4 and anti-CD8-treated FKBP- $-5^{-/}$mice than untreated FKBP- $5^{-/-}$mice $(\mathrm{P}<0.01$; see Figure $4 B$ ). Additionally, our results showed that the levels of gut BAs, the weight of abdomen adipose tissues, and the level of serum total cholesterol were markedly higher in anti-CD4 and anti-CD8-treated FKBP- $5^{-/-}$mice than untreated FKBP $-5^{-/}$mice $(\mathrm{P}<0.01$; see Figure $4 C)$. Further, the results of the phylum bar plot showed that 


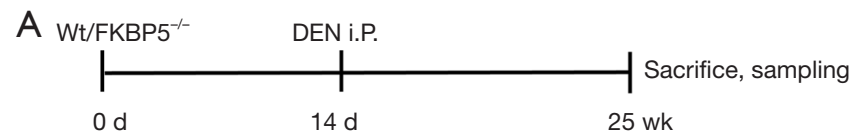

B
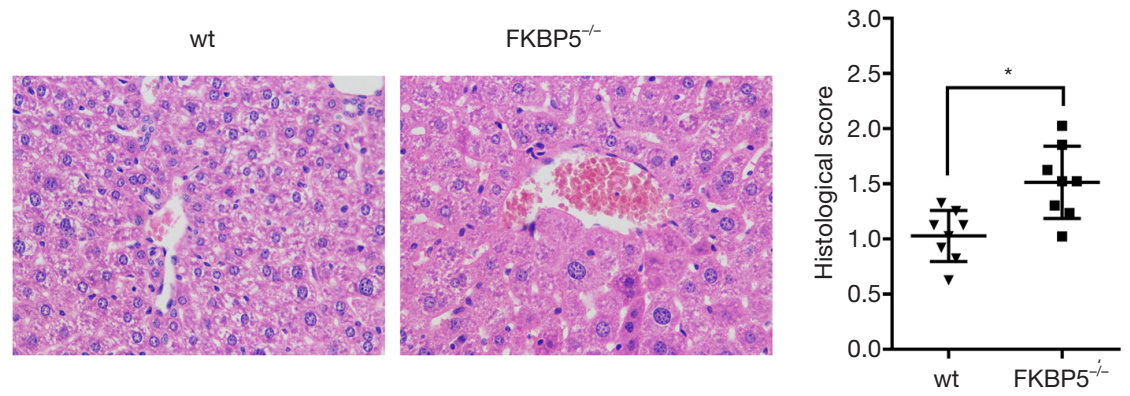

D

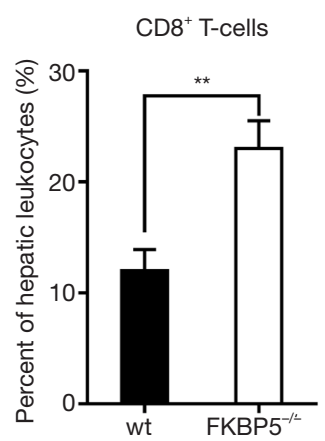

CD4 ${ }^{+}$T-cells

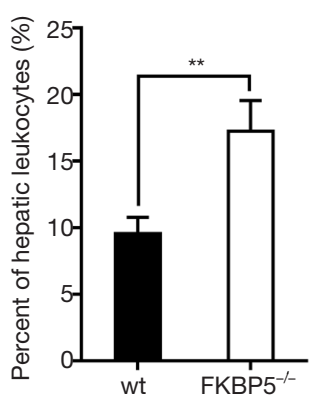

NKT T-cells

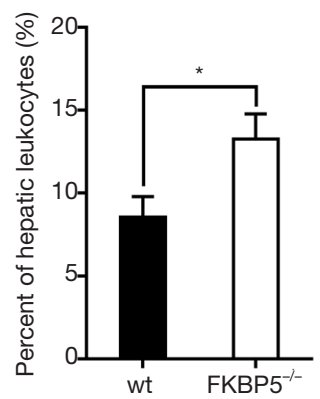

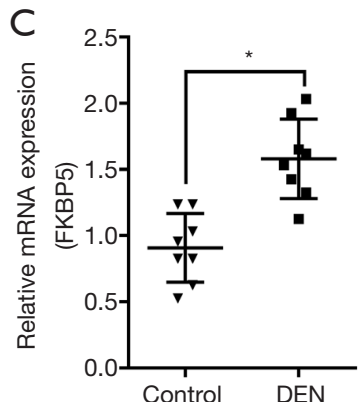

CD4 ${ }^{+}$NKT-cells

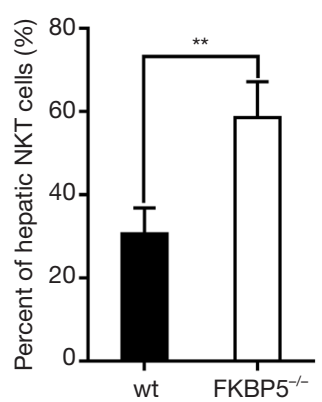

Figure 2 The deficiency of FKBP-5 facilitated cytokine-releasing lymphocytes in HCC. (A) Schematic diagram of WT and FKBP-5 $5^{-1-}$ mice treatment by intraperitoneal injection (i.p.). (B) H\&E staining was applied to assess the histological characteristics of the livers of WT and FKBP- $5^{--}$mice. Magnification, 100×; Scale bar $=100 \mu \mathrm{m}$. The histological score was counted. *, $\mathrm{P}<0.05 v s$. WT mice. (C) A RTqPCR analysis of FKBP-5 was performed in DEN-treated and untreated-WT mice. *, $\mathrm{P}<0.05$ vs. control (DEN-untreated mice) group. (D) Hepatic leukocytes $\left(\mathrm{CD}^{+} \mathrm{T}\right.$ cells, $\mathrm{CD} 4^{+} \mathrm{T}$ cells, NKT cells) and hepatic NKT cells were evaluated by an FACS assay. The percentages were calculated. *, $\mathrm{P}<0.05 ;{ }^{* *}, \mathrm{P}<0.01$ vs. control group.

the abundance of bacteroidales and verrucomicrobiales were decreased, and clostridiales, burkbolderiales and enterobacteriales were increased after anti-CD4 and anti-CD8 treatments in $\mathrm{FKBP}_{-} 5^{--}$mice (see Figure $4 D$ ). Thus, a combination of anti-CD4 and anti-CD8 appears to induce the obesityassociated gut microbial composition.

\section{Discussion}

FKBP-5 is a protein located on the surface membrane of cells that largely contains three domains; that is, two FKBD domains (FK1, and FK2) and one TPR domain (TPR1, TPR2, and TPR3) (35). Previous research has suggested that FKBP-5 is widely expressed in kidney, skeletal muscle, liver, placenta, heart, peripheral lymphocyte, small intestine, ovary, testis, thymus, and lymph node (36-39). Previous studies have found that FKBP-5 enhances cancer cell motility and invasion (40), and FKBP-5 is involved in the development processes of various cancers, including breast cancer, myeloma, prostate cancer, melanoma, pancreatic cancer, and acute lymphoblastic leukemia (19-25). In the present study, we further demonstrated that FKBP5 expression is significantly increased in HCC tissues and cells, which suggests that FKBP-5 might accelerate hepatocellular progression. Notably, the expression trend of FKBP-5 in HCC is consistent with other cancers as identified in previous research.

Previous research has shown that FKBP-5 is an immune macromolecule with various biological functions $(41,42)$, and that FKBP-5 plays a pivotal role in immunoregulation, 
A

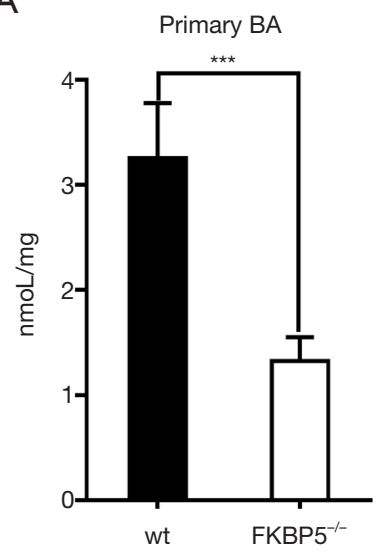

Abdomen adipose tissue

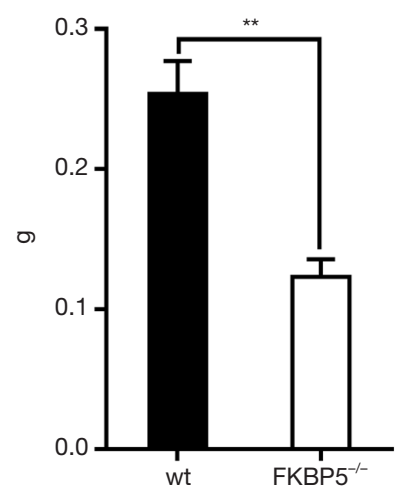

Secondary BA

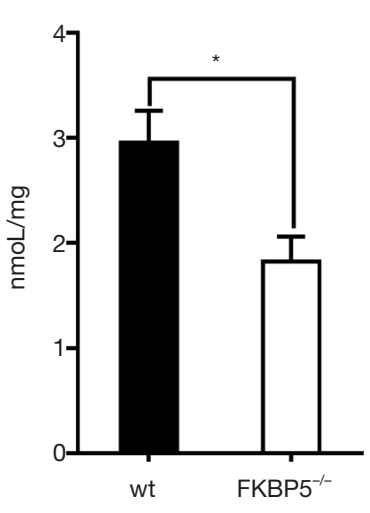

Serum total cholesterol

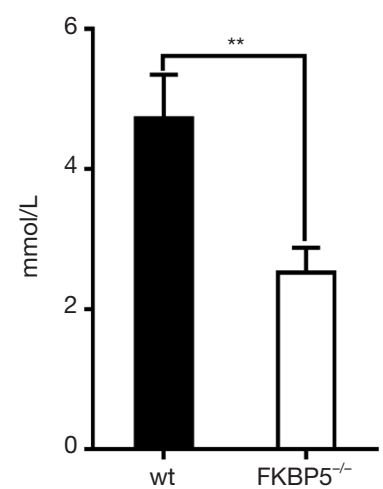

B

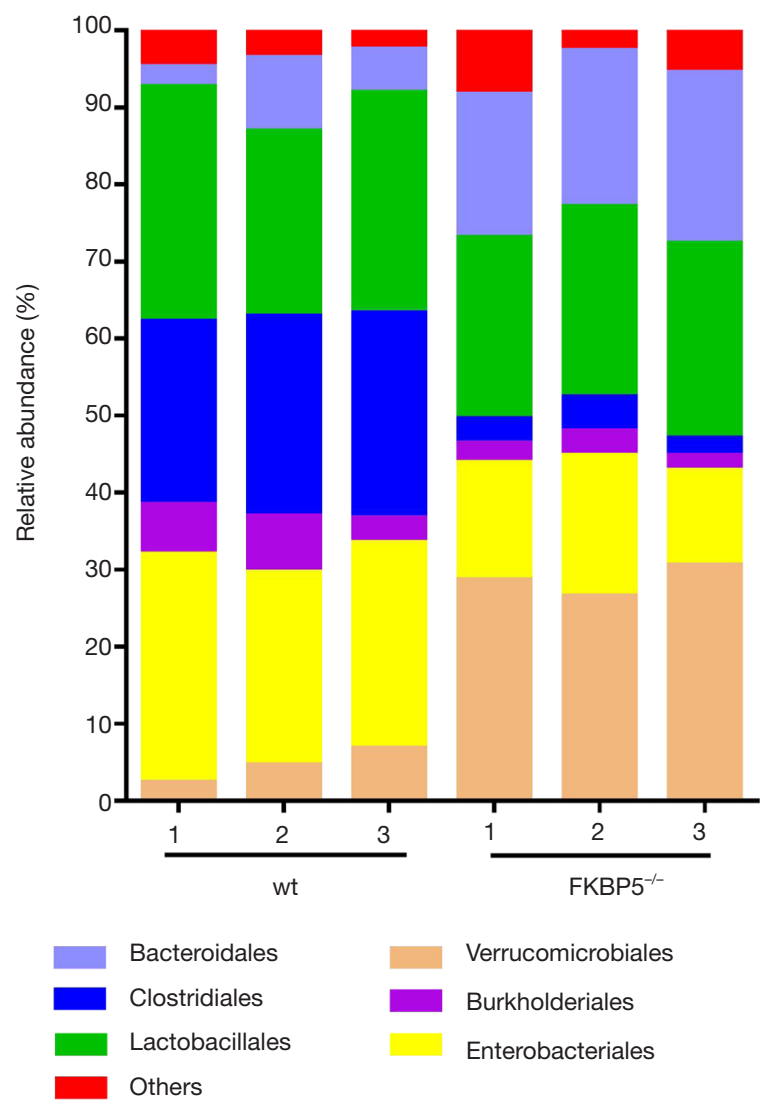

Figure 3 FKBP-5 deletion-mediated gut microbial alterations were related to BAs. (A) The concentrations of primary BAs, secondary BAs, serum total cholesterol, and the weight of the abdomen adipose tissues were measured in $\mathrm{WT}$ and $\mathrm{FKBP}-5^{-/-}$mice. * $\mathrm{P}<0.05 ;{ }^{* *}, \mathrm{P}<0.01$; ***, $\mathrm{P}<0.001$ vs. WT mice. (B) Mean percentage of the total population in gut microbiota in WT and FKBP- $5^{-1-}$ mice.

steroid hormone regulation, depression, carcinogenesis, and drug resistance (12). For example, the combination of immunosuppressor and FKBP-5 appears to inhibit the $\mathrm{Ca}^{2+}$ signaling pathway, and consequently the activation of T cells (43). Thus, FKBP-5 plays a crucial role in immune regulation. Further, other studies have demonstrated that inflammatory microenvironment and immunoregulation are closely related to the progression, angiogenesis and invasion of various tumors, including liver cancer (44-47). However, before this study, the effect of FKBP-5 on an inflammatory microenvironment and immunoregulation in HCC, had not yet been fully elucidated. In this study, we found that FKBP-5 deletion reduces the number and growth of liver tumors and increases the proportions of hepatic leukocytes $\left(\mathrm{CD}^{+} \mathrm{T}\right.$ cells, $\mathrm{CD} 4^{+} \mathrm{T}$ cells, and NKT cells), and hepatic NKT $\left(\mathrm{CD}^{+}{ }^{+} \mathrm{NKT}\right)$ cells. Among them, both natural killer
T cells (NKT cells) and natural killer (NK) cells can exert anti-tumor immune effects, including natural immunity and specific immunity. NK cells are important effector cells that regulate the activation and inhibition of receptors, while NKT cells can eradicate tumor cells (48). $\mathrm{CD}^{+}$and $\mathrm{CD}^{+}$are two major subgroups of $\mathrm{T}$ cells, among which $\mathrm{CD}^{+}$can regulate cytokines, enhance cell-killing ability, regulate humoral immunity, and kill tumor cells (49). Thus, we hypothesized that FKBP-5 deletion would suppress hepatocarcinogenesis by modulating the immune response.

Previous research has suggested that an intestinal flora imbalance often plays a key role in the development of chronic liver disease and HCC (50). Additionally, research has shown that bacterial translocation caused by intestinal leakage can cause infectious complications of advanced liver disease and chronic inflammation (51). For example, 
A

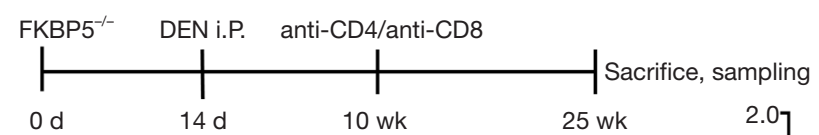

B
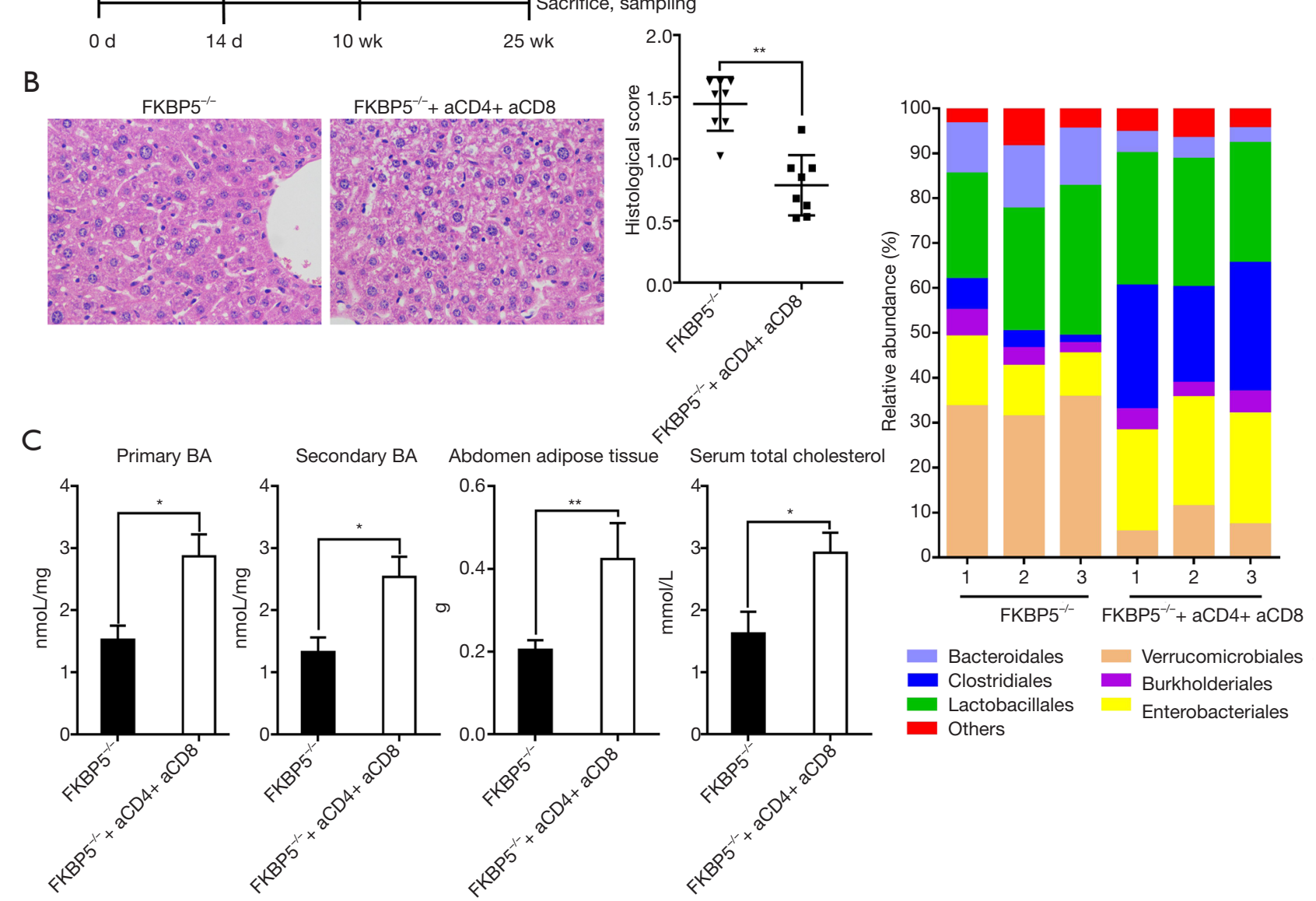

D

Figure 4 Combination of anti-CD4 and anti-CD8 promoted hepatocellular progression by modulating gut microbial community in FKBP$5^{--/}$mice. (A) Timeline of the experimental progress (WT and FKBP- $5^{-/-}$mice). The experiment was divided into the FKBP- $5^{-/-}$group and FKBP- $5^{-/}+$anti-CD4 (aCD4)/anti-CD8 (aCD8) group. (B) The histological features of live were measured by H\&E staining in FKBP$5^{-1-}$ mice and anti-CD4 and anti-CD8-treated FKBP- $5^{-1-}$ mice. The pathologic results were shown; Magnification, 100×; Scale bar $=100 \mu \mathrm{m}$. The histological score was also counted. ${ }^{* *}, \mathrm{P}<0.01 v s$. FKBP- $5^{-1-}$ mice. (C) After the anti-CD4 and anti-CD8 treatments in FKBP- $5^{-/-}$mice, the concentrations of primary BAs, secondary BAs, serum total cholesterol, and the weight of abdomen adipose tissues were examined. *, $\mathrm{P}<0.05 ;{ }^{* *}, \mathrm{P}<0.01 v s$. FKBP- $5^{-/-}$mice. (D) The distribution of gut microbial community in FKBP- $5^{-/-}$mice after treatment with anti-CD4 and anti-CD8.

intestinal microorganisms can regulate the growth of liver cancer by affecting the expression levels of pro-inflammatory and anti-inflammatory cytokines in the liver (52). Another study also found that inflammation in the liver can induce the production of cells that produce immunoglobulin IgA, and promote the formation of HCC by inhibiting the antitumor activity of $\mathrm{CD}^{+} \mathrm{T}$ cells (53). Thus, the anti-tumor immune response in the liver is closely related to intestinal bacteria. Intestinal microbes have also been reported to be closely associated with intestinal inflammation (54), chronic intestinal inflammation (55), metabolic inflammation (56) and liver inflammation (57), etc. Moreover, research has also shown that in patients with chronic liver disease and cirrhosis, bacterial imbalance is manifested by an increase in the number of potential pathogens and a decrease in the number of beneficial bacteria (58). For example, a highfat diet has been shown to lead to an increase of gramnegative bacteria in mice's intestines and a decrease in the ratio of bacteroidetes to firmicutes (59). In our study, we found that FKBP-5 deletion increased bacteroidales and verrucomicrobiales, and decreased clostridiales, burkbolderiales and enterobacteriales in HCC. Thus, it appears that FKBP-5 
deletion could induce gut microbial alterations.

The imbalance of flora is mainly driven by features of end-stage liver disease, such as bile secretion reduction, and changes in the antimicrobial peptides and IgA secreted by the intestine (60). BA refers to a type of cholic acid in bile, which is the end product of cholesterol catabolism (61). Total bile acids (TBAs) include primary BAs and secondary BAs, the generation and metabolism of which are closely related to the liver (62). Currently, TBAs have been used as a sensitive indicator of liver function. In the present study, we found that FKBP-5 deletion could reduce the levels of TBAs, and decrease the weight of abdomen adipose tissues and the level of serum total cholesterol. Thus, we showed that the deficiency of FKBP-5 inhibits the secretion of TBAs and obese phenotypes of mice. In addition, we showed that FKBP-5 deletion-mediated gut microbial alterations are relevant to BAs.

\section{Conclusions}

The present study showed that the deficiency of FKBP5 suppresses hepatocellular progression by increasing the proportions of hepatic leukocytes and inhibiting obesityassociated gut microbial metabolite. Thus, FKBP-5 might serve as a potential therapeutic target of HCC. These results provide novel insights into the complex molecular mechanisms of HCC. However, it should be noted that this study had a number of limitations. For example, it did not examine whether FKBP-5 deletion-induced gut microbial alterations are relevant to the anti-tumor immune response in HCC, or whether there are more regulatory mechanisms in the FKBP5/immune regulation/intestinal bacteria/HCC axis.

\section{Acknowledgments}

Funding: This work was supported by The Sanming Project of Medicine in Shenzhen (No. SZSM201612074), The Guangdong Natural Science Foundation (No. 2018A030313181) and Shenzhen Natural Science Foundation (No. JCYJ20190812161605538).

\section{Footnote}

Reporting Checklist: The authors have completed the ARRIVE reporting checklist. Available at http://dx.doi. org/10.21037/jgo-21-71

Data Sharing Statement: Available at http://dx.doi. org/10.21037/jgo-21-71

Conflicts of Interest: All authors have completed the ICMJE uniform disclosure form (available at: http://dx.doi. org/10.21037/jgo-21-71). The authors have no conflicts of interest to declare.

Ethical Statement: The authors are accountable for all aspects of the work in ensuring that questions related to the accuracy or integrity of any part of the work are appropriately investigated and resolved. The study was conducted in accordance with the Declaration of Helsinki (as revised in 2013). Written and informed consent forms were obtained from all of the participating patients. This study was approved by the Shenzhen Traditional Chinese Medicine Hospital Ethics Committee. The program for the animal experiment was approved by the Institutional Animal Care and Use Committee. In the process of the experiment, the principle of animal welfare was fully considered.

Open Access Statement: This is an Open Access article distributed in accordance with the Creative Commons Attribution-NonCommercial-NoDerivs 4.0 International License (CC BY-NC-ND 4.0), which permits the noncommercial replication and distribution of the article with the strict proviso that no changes or edits are made and the original work is properly cited (including links to both the formal publication through the relevant DOI and the license). See: https://creativecommons.org/licenses/by-nc-nd/4.0/.

\section{References}

1. Forner A, Reig M, Bruix J. Hepatocellular carcinoma. Lancet 2018;391:1301-14.

2. Forner A, Llovet JM, Bruix J. Hepatocellular carcinoma. Lancet 2012;379:1245-55.

3. Stewart B, Wild CP. World cancer report 2014. 2014.

4. Sebastián Domingo JJ, Sánchez Sánchez C. From the intestinal flora to the microbiome. Rev Esp Enferm Dig 2018;110:51-6.

5. Lynch SV, Pedersen O. The Human Intestinal Microbiome in Health and Disease. N Engl J Med 2016;375:2369-79.

6. Garrett WS. Cancer and the microbiota. Science 2015;348:80-6.

7. Yu LX, Schwabe RF. The gut microbiome and liver cancer: mechanisms and clinical translation. Nat Rev Gastroenterol Hepatol 2017;14:527-39.

8. Tripathi A, Debelius J, Brenner DA, et al. The gut-liver 
axis and the intersection with the microbiome. Nat Rev Gastroenterol Hepatol 2018;15:397-411.

9. Caspani G, Kennedy S, Foster JA, et al. Gut microbial metabolites in depression: understanding the biochemical mechanisms. Microb Cell 2019;6:454-81.

10. Albhaisi S, Shamsaddini A, Fagan A, et al. Gut Microbial Signature of Hepatocellular Cancer in Men with Cirrhosis. Liver Transpl 2021. [Epub ahead of print]. doi: 10.1002/ lt.25994.

11. Jiang JW, Chen XH, Ren Z, et al. Gut microbial dysbiosis associates hepatocellular carcinoma via the gut-liver axis. Hepatobiliary Pancreat Dis Int 2019;18:19-27.

12. Harms MB, Birn R, Provencal N, et al. Early life stress, FK506 binding protein 5 gene (FKBP5) methylation, and inhibition-related prefrontal function: A prospective longitudinal study. Dev Psychopathol 2017;29:1895-903.

13. Assimon VA, Southworth DR, Gestwicki JE. Specific binding of tetratricopeptide repeat proteins to heat shock protein 70 (Hsp70) and heat shock protein 90 (Hsp90) is regulated by affinity and phosphorylation. Biochemistry 2015;54:7120-31.

14. Kumar R, Moche M, Winblad B, et al. Combined x-ray crystallography and computational modeling approach to investigate the Hsp90 C-terminal peptide binding to FKBP51. Sci Rep 2017;7:14288.

15. Criado-Marrero M, Rein T, Binder EB, et al. Hsp90 and FKBP51: complex regulators of psychiatric diseases. Philos Trans R Soc Lond B Biol Sci 2018;373:20160532.

16. Han KM, Won E, Sim Y, et al. Influence of FKBP5 polymorphism and DNA methylation on structural changes of the brain in major depressive disorder. Sci Rep 2017;7:42621.

17. Zhang L, Qiu B, Wang T, et al. Loss of FKBP5 impedes adipocyte differentiation under both normoxia and hypoxic stress. Biochem Biophys Res Commun 2017;485:761-7.

18. Zannas AS, Jia M, Hafner K, et al. Epigenetic derepression of FKBP5 by aging and stress contributes to NF-kB-driven inflammation and cardiovascular risk. Proc Natl Acad Sci U S A 2019;116:11370-9.

19. Gillis KA, Zhang Y. Expression analysis of FKBP54 in the diagnosis and treatment of prostate cancer: US, US20090062133 A1[P]. 2005. Available online: https:// www.freepatentsonline.com/20090062133.pdf

20. Kumar P, Mark P, Ward B, et al. Estradiol-regulated expression of the immunophilins cyclophilin 40 and FKBP52 in MCF-7 breast cancer cells. Biochem Biophys Res Commun 2001;284:219-25.

21. Ni L, Yang CS, Gioeli D, et al. FKBP51 promotes assembly of the Hsp90 chaperone complex and regulates androgen receptor signaling in prostate cancer cells. Mol Cell Biol 2010;30:1243-53.
22. Pei H, Li L, Fridley BL, et al. FKBP51 Affects Cancer Cell Response to Chemotherapy by Negatively Regulating Akt. Cancer Cell 2009;16:259-66.

23. D'Arrigo P, Russo M, Guadagno E, et al. Abstract 586: Ionizing radiation-induced PD-L1 upregulation in glioma: a crucial role for the molecular chaperone FKBP5. Cancer Res 2017;77:Abstr 586.

24. Smith E, Palethorpe HM, Ruszkiewicz AR, et al. Androgen Receptor and Androgen-Responsive Gene FKBP5 Are Independent Prognostic Indicators for Esophageal Adenocarcinoma. Dig Dis Sci 2016;61:433-43.

25. Srivastava SK, Bhardwaj A, Arora S, et al. Interleukin-8 is a key mediator of FKBP51-induced melanoma growth, angiogenesis and metastasis. Br J Cancer 2015;112:1772-81.

26. Sidibeh CO, Pereira MJ, Abalo XM, et al. FKBP5 expression in human adipose tissue: potential role in glucose and lipid metabolism, adipogenesis and type 2 diabetes. Endocrine 2018;62:116-28.

27. Warrier M. Role of FKBP5 1 and FKBP52 in Glucocorticoid Receptor Regulated Metabolism. 2008. Available online: http://europepmc.org/article/ETH/3669

28. Zannas AS, Balsevich G, Gassen NC, et al. The emerging role of FKBP5 in the regulation of metabolism and body weight. Surg Obes Relat Dis 2016;12:1560-1.

29. Stechschulte LA, Qiu B, Warrier M, et al. FKBP51 Null Mice Are Resistant to Diet-Induced Obesity and the PPARI ${ }^{3}$ Agonist Rosiglitazone. Endocrinology 2016;157:3888-900.

30. Bajaj M, Suraamornkul S, Hardies LJ, et al. Effects of peroxisome proliferator-activated receptor (PPAR)alpha and PPAR-gamma agonists on glucose and lipid metabolism in patients with type 2 diabetes mellitus. Diabetologia 2007;50:1723-31.

31. Hallundbæk Mikkelsen K, Nielsen MF, Tvede M, et al. Gut microbiota may have influence on glucose and lipid metabolism. Ugeskr Laeger 2013;175:2785-8.

32. Li JJ, Luo J, Lu JN, et al. Relationship between TRAF6 and deterioration of HCC: an immunohistochemical and in vitro study. Cancer Cell Int 2016;16:76.

33. Lin F, Zhang P, Zuo Z, et al. Thioredoxin-1 promotes colorectal cancer invasion and metastasis through crosstalk with S100P. Cancer Lett 2017;401:1-10.

34. Mossanen JC, Krenkel O, Ergen C, et al. Chemokine (C-C motif) receptor 2-positive monocytes aggravate the early phase of acetaminophen-induced acute liver injury. Hepatology 2016;64:1667-82.

35. Matosin N, Halldorsdottir T, Binder EB. Understanding the Molecular Mechanisms Underpinning Gene by Environment Interactions in Psychiatric Disorders: The FKBP5 Model. Biol Psychiatry 2018;83:821-30. 
36. Zannas AS, Wiechmann T, Gassen NC, et al. Gene-StressEpigenetic Regulation of FKBP5: Clinical and Translational Implications. Neuropsychopharmacology 2016;41:261-74.

37. Ising M, Maccarrone G, Bruckl T, et al. FKBP5 Gene Expression Predicts Antidepressant Treatment Outcome in Depression. Int J Mol Sci 2019;20:485.

38. Lieberman R, Kranzler HR, Levine ES, et al. Examining FKBP5 mRNA expression in human iPSC-derived neural cells. Psychiatry Res 2017;247:172-81.

39. Yeo S, Enoch MA, Gorodetsky E, et al. The influence of FKBP5 genotype on expression of FKBP5 and other glucocorticoid-regulated genes, dependent on trauma exposure. Genes Brain Behav 2017;16:223-32.

40. Takaoka M, Ito S, Miki Y, et al. FKBP51 regulates cell motility and invasion via RhoA signaling. Cancer Sci 2017;108:380-9.

41. Juszczak GR, Stankiewicz AM. Glucocorticoids, genes and brain function. Prog Neuropsychopharmacol Biol Psychiatry 2018;82:136-68.

42. Wiechmann T, Roh S, Sauer S, et al. Identification of dynamic glucocorticoid-induced methylation changes at the FKBP5 locus. Clin Epigenetics 2019;11:83.

43. Gant JC, Blalock EM, Chen KC, et al. FK506-Binding Protein 12.6/1b, a Negative Regulator of [Ca2+], Rescues Memory and Restores Genomic Regulation in the Hippocampus of Aging Rats. J Neurosci 2018;38:1030-41.

44. Ben-Baruch A. Host microenvironment in breast cancer development: inflammatory cells, cytokines and chemokines in breast cancer progression: reciprocal tumor-microenvironment interactions. Breast Cancer Res 2003;5:31-6.

45. de Visser KE, Coussens LM. The inflammatory tumor microenvironment and its impact on cancer development. Contrib Microbiol 2006;13:118-37.

46. Sfanos KS, Yegnasubramanian S, Nelson WG, et al. The inflammatory microenvironment and microbiome in prostate cancer development. Nat Rev Urol 2018;15:11-24.

47. Matter MS, Marquardt JU, Andersen JB, et al. Oncogenic driver genes and the inflammatory microenvironment dictate liver tumor phenotype. Hepatology 2016;63:1888-99.

48. Pellicci DG, Hammond KJ, Uldrich AP, et al. A natural killer T (NKT) cell developmental pathway ilnvolving a thymus-dependent NK1.1(-)CD4(+) CD1d-dependent precursor stage. J Exp Med 2002;195:835-44.

49. Ge Q, Chen WF. Phenotypic identification of the subgroups of murine T-cell receptor alphabeta+ CD4+ CD8- thymocytes and its implication in the late stage of thymocyte development. Immunology 1999;97:665-71.

50. Zhang D, Hao X, Xu L, et al. Intestinal flora imbalance promotes alcohol-induced liver fibrosis by the TGF $\beta /$ smad signaling pathway in mice. Oncol Lett 2017;14:4511-6.

51. Szabo G, Petrasek J. Inflammasome activation and function in liver disease. Nat Rev Gastroenterol Hepatol 2015;12:387-400.

52. Loo TM, Kamachi F, Watanabe Y, et al. Gut microbiota promotes obesity-associated liver cancer through PGE2mediated suppression of antitumor immunity. Cancer Discov 2017;7:522-38.

53. Shalapour S, Lin XJ, Bastian IN, et al. Inflammationinduced IgA+ cells dismantle anti-liver cancer immunity. Nature 2017;5 51:340-5.

54. Amoroso C, Perillo F, Strati F, et al. The Role of Gut Microbiota Biomodulators on Mucosal Immunity and Intestinal Inflammation. Cells 2020;9:1234.

55. Serra D, Almeida LM, Dinis TCP. The Impact of Chronic Intestinal Inflammation on Brain Disorders: the MicrobiotaGut-Brain Axis. Mol Neurobiol 2019;56:6941-51.

56. Tilg H, Zmora N, Adolph TE, et al. The intestinal microbiota fuelling metabolic inflammation. Nat Rev Immunol 2020;20:40-54.

57. Wang W, Zhai S, Xia Y, et al. Ochratoxin A induces liver inflammation: involvement of intestinal microbiota. Microbiome 2019;7:151.

58. Zhang YJ, Li S, Gan RY, et al. Impacts of gut bacteria on human health and diseases. Int J Mol Sci 2015;16:7493-519.

59. Onishi JC, Campbell S, Moreau M, et al. Bacterial communities in the small intestine respond differently to those in the caecum and colon in mice fed low-and highfat diets. Microbiology (Reading) 2017;163:1189-97.

60. Lin YL, Ip PP, Liao F. CCR6 Deficiency Impairs IgA Production and Dysregulates Antimicrobial Peptide Production, Altering the Intestinal Flora. Front Immunol 2017;8:805.

61. Chiang JYL. Bile acid metabolism and signaling in liver disease and therapy. Liver Res 2017;1:3-9.

62. Janssen AW, Houben T, Katiraei S, et al. Modulation of the gut microbiota impacts nonalcoholic fatty liver disease: a potential role for bile acids. J Lipid Res 2017;58:1399-416.

(English Language Editor: L. Huleatt)

Cite this article as: Zhang C, Cui X, Feng L, Han Z, Peng D, Fu W, Xing Y. The deficiency of FKBP-5 inhibited hepatocellular progression by increasing the infiltration of distinct immune cells and inhibiting obesity-associated gut microbial metabolite. J Gastrointest Oncol 2021;12(2):711-721. doi: 10.21037/jgo-21-71 